\title{
Linear Prediction Approach to Oversampling Parameter Estimation for Multiple Complex Sinusoids
}

\author{
Zhenhua ZHOU and H. C. SO \\ Department of Electronic Engineering \\ City University of Hong Kong, Tat Chee Avenue, Kowloon, Hong Kong \\ Email : zhenhzhou2@student.cityu.edu.hk
}

Keywords : Sinusoidal parameter estimation, linear prediction, weighted least squares, oversampling, decimation

\begin{abstract}
The problem of oversampling parameter estimation for noisy sinusoidal signals is addressed. We first extend the weighted least squares (WLS) approach to the complex sinusoids. Then the oversampling weighted least squares (OSWLS) estimator is proposed based on data decimation. Estimation performance of the OSWLS method is analyzed via theoretical and simulation studies. Results are also compared to those of the WLS and decimative unitary ESPRIT methods as well as Cramér-Rao lower bound.
\end{abstract}

\section{Introduction}

Spectral estimation from a finite record of a stationary data sequence is a classical but active problem, finding applications in a wide range of areas such as vibration monitoring, astronomy and speech analysis [1]. It can be classified into two categories, namely, nonparametric and parametric. The latter, which assumes that the signal satisfies a generating model with known functional form, is popular in practice for its high frequency resolution. To overcome the demanding computational requirement of the optimum parametric estimators such as maximum likelihood (ML) [2], nonlinear least squares (NLS) [3], kinds of techniques have been proposed based on the linear prediction property of sinusoidal signals [4] - [7]. In particular, [8] devises the weighted linear squares (WLS) frequency estimator with good computational efficiency and optimum estimation accuracy. Nevertheless, the WLS approach in [8] only focuses on the real sinusoids, and there is a need to extend it to a more general situation of multiple complex sinusoids.

During the recent decades, numerous research has been performed for frequency estimation. But limited attention is paid to oversampling line spectra analysis. In signal processing, oversampling is the process of sampling a signal with a sampling frequency significantly higher than the Nyquist frequency of the signal being sampled. In 1-bit quantization spectral estimation [9], it is used to compress the spectrum, and to avoid the singular frequency which incurs high estimation error. In [10], oversampling is utilized to obtain 
more data in a fixed duration, and is expected to improve the estimation accuracy according to Cramér-Rao lower bound (CRLB) [11] analysis. However, to the best of our knowledge, no further exploration in this direction is made to devise a practical frequency estimator with better performance.

In this paper, we first extend the WLS approach to the case of multiple complex sinusoids. The motivation of this paper is to investigate whether the WLS estimation accuracy could be improved with oversampling in practice. Through oversampling, the signal's spectrum is compressed towards the origin due to the higher sampling rate. Meanwhile, more samples are available within a fixed duration. Accordingly, there are two problems occurring in spectral estimation: i) the signal's angular frequencies become closer, which reduces frequency resolution; ii) the computational complexity is increased. To tackle these problems, the oversampling WLS (OSWLS) frequency estimator with decimation is proposed in this paper. The major contributions of this paper are summarized as: i) extension of the WLS estimator to the multiple complex sinusoids; ii) practical implementation of the OSWLS method; and iii) derivation of the statistical properties of the OSWLS.

The rest of this paper is organized as follows. In Section 2, the problem of oversampling spectral estimation for noisy multiple-tone sinusoidal signals is formulated and the OSWLS method is developed. In Section 3, theoretical analysis of the statistical properties of the OSWLS is provided, including the frequency resolution, computational complexity and estimation variance. Simulation results are presented in Section 4 to evaluate and verify the performance of the proposed approach. Finally, summary is drawn in Section 5.

\section{Problem Formulation and Algorithm Development}

\subsection{Problem Formulation}

The problem of multiple complex sinusoidal frequency estimation is formulated as follows. Given the sampled noisy measurements

$$
x(n)=s(n)+q(n), \quad n=1, \cdots, N,
$$

where

$$
s(n)=\sum_{l=1}^{L} \alpha_{l} e^{j\left(2 \pi f_{l} n / f_{S}+\phi_{l}\right)},
$$

with $\alpha_{l}, f_{l} \in\left(0, f_{S} / 2\right), \phi_{l} \in[0,2 \pi)$ being the unknown deterministic parameters which denote the amplitude, frequency, initial phase of the $l$-th complex-valued sinusoid, $f_{S}$ the known sampling rate, $N$ the length of the data sequence; $q(n)$ is an additive white Gaussian noise with unknown variance $\sigma_{q}^{2}$. Furthermore, it is assumed that the number of sinusoids $L$ is known a priori. Here the angular frequencies $\omega_{l}=2 \pi f_{l} / f_{S}(0<$ $\left.\omega_{l}<\pi\right)$ are in main concern, for they are nonlinear in the observed data. Once their estimates are obtained, the remaining parameters can be estimated as a linear least-squares (LS) solution [12].

From (2), it is obvious that the higher the sampling rate $f_{S}$ is, the smaller the angular frequencies $\omega_{l}$ are and thus the narrower spectrum, which causes the problems of frequency resolution and computational complexity. The next subsections focus on circumventing them, and achieving high accuracy simultaneously. 


\subsection{Extension of WLS Approach to Complex Sinusoids}

In [8], the linear prediction (LP) equation is established, and the LP coefficients are estimated by the minimization of the weighted LP error. Then the frequency estimates are obtained from the relationship between the frequencies and LP coefficients. Nonetheless, [8] only considers the real sinusoids. Indeed, there have been some literatures about the WLS-based frequency estimation such as [13], [14], which are applied to the complex case. But they only consider the general damped sinusoidal estimation, and impose no unity-magnitude constraint on the pole estimates. Thus, these methods cannot provide optimum estimation accuracy in undamped complex sinusoidal estimation.

To overcome this problem, the forward-backward (FB) technique is utilized to keep the LP coefficients real and the pole estimates on the unit circle. Accordingly, the forward and backward LP equations are established as:

$$
\begin{array}{ll}
\sum_{i=0}^{L} a_{i} s(n-i)=0, & a_{0}=1, \quad n=L+1, L+2, \cdots, N, \\
\sum_{i=0}^{L} a_{i} s(n+i)=0, \quad a_{0}=1, & n=1,2, \cdots, N-L .
\end{array}
$$

Combining (3) and (4), the new LP equation is

$$
\sum_{i=0}^{L-1} a_{i}[s(n+i)+s(n+2 L-i)]+a_{L} s(n+L)=0, \quad n=1,2, \cdots, N-2 L,
$$

where $a_{i}$ are the LP coefficients to be estimated from the noisy data by solving the following WLS problem

$$
\hat{\mathbf{a}}=\arg \min _{\tilde{\mathbf{a}} \in \mathbb{R}^{L}} f(\tilde{\mathbf{a}})=\mathbf{e}^{H} \mathbf{W e},
$$

where

$$
\mathbf{e}=\mathbf{X} \tilde{\mathbf{a}}-\mathbf{b}
$$

$$
\begin{aligned}
& \mathbf{X}=\left(\begin{array}{cccc}
x(2)+x(2 L) & \cdots & x(L)+x(L+2) & x(L+1) \\
x(3)+x(2 L+1) & \cdots & x(L+1)+x(L+3) & x(L+2) \\
\vdots & \ddots & \vdots & \vdots \\
x(N-2 L+1)+x(N-1) & \cdots & x(N-L-1)+x(N-L+1) & x(N-L)
\end{array}\right), \\
& \mathbf{b}=-[x(1)+x(2 L+1), \quad x(2)+x(2 L+2), \quad \cdots, \quad x(N-2 L)+x(N)]^{T},
\end{aligned}
$$

and $\tilde{\mathbf{a}}=\left[\begin{array}{llll}\tilde{a}_{1}, & \tilde{a}_{2}, & \cdots, & \tilde{a}_{L}\end{array}\right]^{T}$ is the optimization variable for the coefficient vector $\mathbf{a}=$ $\left[\begin{array}{llll}a_{1}, & a_{2}, & \cdots & a_{L}\end{array}\right]^{T}$. Since a satisfies the forward and backward LP equations simultaneously, the LP coefficients are real. The optimum weighting matrix $\mathbf{W}$ is obtained from Markov estimate [15]:

$$
\mathbf{W}=\sigma_{q}^{2}\left[\left.E\left\{\mathbf{e e}^{H}\right\}\right|_{\tilde{\mathbf{a}}=\mathbf{a}}\right]^{-1}=\left(\mathbf{A} \mathbf{A}^{H}\right)^{-1},
$$


where

$$
\begin{aligned}
\mathbf{A} & =\operatorname{Toeplitz}\left(\left[1, \mathbf{0}_{1 \times(N-2 L-1)}\right]^{T},\left[1, a_{1}, \cdots, a_{L}, \cdots, a_{1}, 1, \mathbf{0}_{1 \times(N-2 L-1)}\right]\right) \\
& =\left[\begin{array}{ccccccccccccccccc}
1 & a_{1} & \cdots & a_{L} & \cdots & a_{1} & 1 & 0 & 0 & \cdots & 0 & 0 & \cdots & 0 & \cdots & 0 & 0 \\
0 & 1 & a_{1} & \cdots & a_{L} & \cdots & a_{1} & 1 & 0 & \cdots & 0 & 0 & \cdots & 0 & \cdots & 0 & 0 \\
\vdots & \vdots & \vdots & \ddots & \vdots & \ddots & \vdots & \vdots & \vdots & \ddots & \vdots & \vdots & \vdots & \vdots & \ddots & \vdots & \vdots \\
0 & 0 & 0 & \cdots & 0 & \cdots & 0 & 0 & 0 & \cdots & 1 & a_{1} & \cdots & a_{L} & \cdots & a_{1} & 1
\end{array}\right] .
\end{aligned}
$$

Assuming that the weighting matrix $\mathbf{W}$ is independent of $\mathbf{a}$, and considering that the LP coefficients are real, the solution to (6) is

$$
\hat{\mathbf{a}}=\left(\mathbf{X}_{r}^{T} \mathbf{W} \mathbf{X}_{r}+\mathbf{X}_{i}^{T} \mathbf{W} \mathbf{X}_{i}\right)^{-1}\left(\mathbf{X}_{r}^{T} \mathbf{W} \mathbf{b}_{r}+\mathbf{X}_{i}^{T} \mathbf{W} \mathbf{b}_{i}\right),
$$

where $\mathbf{X}_{r}$ and $\mathbf{X}_{i}$ are the real and imaginary parts of $\mathbf{X}$, respectively. The notations of $\mathbf{b}_{r}$ and $\mathbf{b}_{i}$ are similar. It is worth mentioning that we can also solve $\hat{\mathbf{a}}$ directly by $\hat{\mathbf{a}}=\left(\mathbf{X}^{H} \mathbf{W} \mathbf{X}\right)^{-1} \mathbf{X}^{H} \mathbf{W} \mathbf{b}$. What we do in (12) is indeed to convert the complex multiplication into the real multiplication, which saves half of the computation. Note that $\mathbf{a}$ is required in computing $\mathbf{W}$, while it is not available $a$ priori, the estimated value of $\mathbf{a}$ is used to construct the weighting matrix $\mathbf{W}$. As a result, $\hat{\mathbf{a}}$ is obtained in a relaxation procedure as follows:

- Step 1. Determine the initial estimates $\hat{\mathbf{a}}_{0}$ from (12) by setting $\mathbf{W}$ as an identity matrix.

- Step 2. Use $\hat{\mathbf{a}}_{0}$ to construct the weighting matrix $\mathbf{W}$, and find an updated estimate $\hat{\mathbf{a}}$ for $\hat{\mathbf{a}}_{0}$.

- Step 3. Repeat Step 2 until satisfactory performance is attained, and then the final estimate $\hat{\mathbf{a}}$ is obtained.

It is proved in [16] that the roots of the following symmetric real-coefficient LP equation lie on the unit circle:

$$
\sum_{i=0}^{L-1} a_{i}\left(z^{i}+z^{2 L-i}\right)+a_{L} z^{L}=0,
$$

and the angular frequencies $\omega_{l}$ can be obtained from the root phases [17]. Note that (13) also produces additional $L$ image frequencies $\omega_{l}^{\prime}=-\omega_{l}, l=1,2, \cdots, L$. In addition, the simulation results in [8] show that the WLS approach performs well when the angular frequencies $\omega_{l}(l=1,2, \cdots, L)$ lie in the interval $[0.1 \pi, 0.9 \pi]$. Hence, the signal has to be sampled at least at the base frequency $f_{B}=2.5 f_{N}$ for the WLS estimation, where $f_{N}=\max _{l}\left\{f_{l}\right\}$ is the Nyquist frequency; and shifted in angular frequency by $0.1 \pi$. In practice, the oversampling rate is taken as the integer multiple of $f_{B}$. In such sampling setting, the frequency estimates $\hat{\omega}_{l}$ are derived as the phases of the $L$ roots of the estimated version of (13) lying in the interval $(0, \pi)$, and the signal frequency estimates $\hat{f}_{l}=\hat{\omega}_{l} \cdot f_{S} / 2 \pi$. For convenience and straightforwardness, the frequency shifting is neglected here. 


\subsection{Implementation of WLS Oversampling Spectral Estimation}

Oversampling is utilized here to get more data points, and is expected to provide more accurate frequency estimation in a fixed observation period. Generally, the sampling rate is susceptible to the sampler performance and the special application. For example, in the 1-bit quantization frequency estimation, a sampling rate larger than 4 times the Nyquist frequency is required [9]. It has been proved [18] that the CRLB for angular frequency estimation decreases at the rate of $\mathcal{O}\left(N^{-3}\right)$ asymptotically. Furthermore, it can be easily derived from [18] that the asymptotic condition still holds when the values of the frequencies change in the presence of white Gaussian noise. As a result, the CRLBs for the signal frequency estimators $\hat{f}_{l}$ in a fixed duration $T$ decrease at the rate of $\mathcal{O}\left(N^{-1}\right)$ asymptotically, that is, the CRLBs decrease by about $10 \mathrm{~dB}$ for a 10-time increase in sampling rate. Thus if the conditions allow, an oversampling rate over 10 times the base frequency $f_{B}$ is expected for 1-bit precision improvement. However, the high sampling rate produces two problems, namely, frequency resolution and computational load. Especially the former is critical. If the estimator cannot resolve two closely-spaced frequencies, incorrect frequency estimation will be resulted in.

In [19], [20], it is pointed out that the fundamental difficulty in the resolution of adjacent frequencies is a numerical problem regarding data matrix conditioning. Closely spaced frequencies cause the matrix to be ill-conditioned. For a general LS problem

$$
\hat{\mathbf{y}}=\arg \min _{\tilde{\mathbf{y}} \in \mathbb{R}^{n}} f(\tilde{\mathbf{y}})=(\mathbf{F} \tilde{\mathbf{y}}-\mathbf{x})^{T}(\mathbf{F} \tilde{\mathbf{y}}-\mathbf{x})=\mathbf{F}^{\dagger} \mathbf{x}
$$

with $\mathbf{F} \in \mathbb{R}^{m \times n}$ being full column rank, $\mathbf{x} \in \mathbb{R}^{m}$, and their perturbation $\Delta \mathbf{F}, \Delta \mathbf{x}$, the resultant relative error of $\hat{\mathbf{y}}$ is proved to be within [21]:

$$
\frac{\|\Delta \hat{\mathbf{y}}\|_{2}}{\|\hat{\mathbf{y}}\|_{2}} \leq \frac{k(\mathbf{F})}{1-\eta}\left(\epsilon_{\mathbf{F}}+\epsilon_{\mathbf{x}}+\epsilon_{\mathbf{F}} k(\mathbf{F}) \frac{\|\mathbf{r}\|_{2}}{\|\mathbf{x}\|_{2}}\right)+\epsilon_{\mathbf{F}} k(\mathbf{F}),
$$

if

$$
\begin{gathered}
\eta<1, \\
\operatorname{rank}(\mathbf{F}+\Delta \mathbf{F})=\operatorname{rank}(\mathbf{F}),
\end{gathered}
$$

where $\|\cdot\|_{2}$ means the matrix/vector 2-norm, $\mathbf{r}=\mathbf{x}-\mathbf{F} \hat{\mathbf{y}}, \epsilon_{\mathbf{F}}=\|\Delta \mathbf{F}\|_{2} /\|\mathbf{F}\|_{2}, \epsilon_{\mathbf{x}}=\|\Delta \mathbf{x}\|_{2} /\|\mathbf{x}\|_{2}, \eta=k(\mathbf{F}) \epsilon_{\mathbf{F}}$, and $k(\mathbf{F})=\|\mathbf{F}\|_{2} \cdot\left\|\mathbf{F}^{\dagger}\right\|_{2}$ is the condition number, that is, the ratio of the largest singular value of $\mathbf{F}$ to the smallest one.

Considering that the weighting matrix $\mathbf{W}$ is Hermitian positive definite, the corresponding WLS problem

$$
\hat{\mathbf{y}}=\arg \min _{\tilde{\mathbf{y}} \in \mathbb{R}^{n}} f(\tilde{\mathbf{y}})=(\mathbf{F} \tilde{\mathbf{y}}-\mathbf{x})^{T} \mathbf{W}(\mathbf{F} \tilde{\mathbf{y}}-\mathbf{x})
$$

is equivalent to the LS problem in the following form

$$
\hat{\mathbf{y}}=(\mathbf{B F})^{\dagger}(\mathbf{B x})
$$

where $\mathbf{B}^{T} \mathbf{B}=\mathbf{W}$. It can be proved that $[22]$

$$
k(\mathbf{B F}) \leq k(\mathbf{B}) \cdot k(\mathbf{F})
$$


In order to reduce the condition number, it is proposed to decimate $x(n)$ to several subsequences $x_{i}(n)$ with a lower sampling rate, and then construct data matrix and vector for each subsequence, stack them together, solve the WLS estimate of $\mathbf{a}$. Since adjacent angular frequencies are set apart by decimation, lower condition number is expected, and thus higher accuracy. The above methodology is termed as the OSWLS frequency estimator, and detailed as follows.

Decimate the signal $x(n)$ with factor $D$, and the subsequences are

$$
x_{i}(n)=x(D(n-1)+i),
$$

for $i=1,2, \cdots, D$, and $n=1,2, \cdots, N^{\prime}$ where $N^{\prime}$ is the largest integer less than $N / D$. Following the algorithm development in the last subsection, for each segment $x_{i}(n), i=1,2, \cdots, D$, the LP error vector becomes

$$
\mathbf{e}_{i}=\mathbf{X}_{i} \tilde{\mathbf{a}}^{\prime}-\mathbf{b}_{i}
$$

where

$$
\begin{aligned}
& \mathbf{X}_{i}=\left(\begin{array}{cccc}
x_{i}(2)+x_{i}(2 L) & \cdots & x_{i}(L)+x_{i}(L+2) & x_{i}(L+1) \\
x_{i}(3)+x_{i}(2 L+1) & \cdots & x_{i}(L+1)+x_{i}(L+3) & x_{i}(L+2) \\
\vdots & \ddots & \vdots & \vdots \\
x_{i}\left(N^{\prime}-2 L+1\right)+x_{i}\left(N^{\prime}-1\right) & \cdots & x_{i}\left(N^{\prime}-L-1\right)+x_{i}\left(N^{\prime}-L+1\right) & x_{i}\left(N^{\prime}-L\right)
\end{array}\right), \\
& \mathbf{b}_{i}=-\left[x_{i}(1)+x_{i}(2 L+1), \quad x_{i}(2)+x_{i}(2 L+2), \quad \cdots, \quad x_{i}\left(N^{\prime}-2 L\right)+x_{i}\left(N^{\prime}\right)\right]^{T},
\end{aligned}
$$

and $\tilde{\mathbf{a}}^{\prime}=\left[\begin{array}{cccc}\tilde{a}_{1}^{\prime}, & \tilde{a}_{2}^{\prime}, & \cdots, & \tilde{a}_{L}^{\prime}\end{array}\right]^{T}$ is the optimization variable for the decimated LP coefficient vector $\mathbf{a}^{\prime}=\left[\begin{array}{llll}a_{1}^{\prime}, & a_{2}^{\prime}, & \cdots & a_{L}^{\prime}\end{array}\right]^{T}$. Accordingly, the WLS cost function for $x_{i}(n)$ is

$$
f_{i}\left(\tilde{\mathbf{a}}^{\prime}\right)=\mathbf{e}_{i}^{H} \mathbf{W}_{S} \mathbf{e}_{i}
$$

where

$$
\begin{gathered}
\mathbf{W}_{S}=\left(\mathbf{A}_{S} \mathbf{A}_{S}^{H}\right)^{-1} \\
\mathbf{A}_{S}=\operatorname{Toeplitz}\left(\left[1, \mathbf{0}_{1 \times\left(N^{\prime}-2 L-1\right)}\right]^{T},\left[1, a_{1}^{\prime}, \cdots, a_{L}^{\prime}, \cdots, a_{1}^{\prime}, 1, \mathbf{0}_{1 \times\left(N^{\prime}-2 L-1\right)}\right]\right) .
\end{gathered}
$$

Stacking the $D$ WLS cost functions together, the coefficient vector $\mathbf{a}^{\prime}$ is estimated by the following minimization

$$
\hat{\mathbf{a}}^{\prime}=\arg \min _{\tilde{\mathbf{a}}^{\prime} \in \mathbb{R}^{L}} f\left(\tilde{\mathbf{a}}^{\prime}\right)=\left[\begin{array}{llll}
\mathbf{e}_{1}^{H} & \mathbf{e}_{2}^{H} & \cdots & \mathbf{e}_{D}^{H}
\end{array}\right] \mathbf{W}_{D}\left[\begin{array}{llll}
\mathbf{e}_{1}^{H} & \mathbf{e}_{2}^{H} & \cdots & \mathbf{e}_{D}^{H}
\end{array}\right]^{H},
$$

where $\mathbf{W}_{D}=\operatorname{blkdiag}\left(\mathbf{W}_{S}, \cdots, \mathbf{W}_{S}\right)$ with blkdiag(.) denoting block diagonal matrix, and $\hat{\mathbf{a}}^{\prime}$ stands for the OSWLS estimate of $\mathbf{a}^{\prime}$. Denote the real and imaginary parts of $\mathbf{X}_{i}, \mathbf{b}_{i}$ as $\mathbf{X}_{r i}, \mathbf{b}_{r i}$ and $\mathbf{X}_{i i}, \mathbf{b}_{i i}$, respectively, $\hat{\mathbf{a}}^{\prime}$ is computed as

$$
\hat{\mathbf{a}}^{\prime}=\left(\sum_{i=1}^{D} \mathbf{X}_{r i}^{T} \mathbf{W}_{S} \mathbf{X}_{r i}+\sum_{i=1}^{D} \mathbf{X}_{i i}^{T} \mathbf{W}_{S} \mathbf{X}_{i i}\right)^{-1}\left(\sum_{i=1}^{D} \mathbf{X}_{r i}^{T} \mathbf{W}_{S} \mathbf{b}_{r i}+\sum_{i=1}^{D} \mathbf{X}_{i i}^{T} \mathbf{W}_{S} \mathbf{b}_{i i}\right) .
$$


Suppose that the oversampling rate is $M f_{B}$ where $M$ is an integer. Then comparing (18), (19) and (28), (29), it is derived that the relative coefficient estimation error with $D$-decimation is:

$$
\begin{aligned}
\frac{\left\|\Delta \mathbf{a}^{\prime}\right\|_{2}}{\left\|\mathbf{a}^{\prime}\right\|_{2}} & \leq \frac{k\left(\mathbf{B}_{C} \mathbf{S}_{C}\right)}{1-k\left(\mathbf{B}_{C} \mathbf{S}_{C}\right) \epsilon_{1}}\left(\epsilon_{1}+\epsilon_{2}\right)+k\left(\mathbf{B}_{C} \mathbf{S}_{C}\right) \epsilon_{1} \\
& \leq \frac{k\left(\mathbf{B}_{C}\right) k\left(\mathbf{S}_{C}\right)}{1-k\left(\mathbf{B}_{C}\right) k\left(\mathbf{S}_{C}\right) \epsilon_{1}}\left(\epsilon_{1}+\epsilon_{2}\right)+k\left(\mathbf{B}_{C}\right) k\left(\mathbf{S}_{C}\right) \epsilon_{1},
\end{aligned}
$$

where $\epsilon_{1}=\left\|\mathbf{B}_{C} \mathbf{Q}_{C}\right\|_{2} /\left\|\mathbf{B}_{C} \mathbf{S}_{C}\right\|_{2}, \epsilon_{2}=\left\|\mathbf{B}_{C} \mathbf{q}_{C}\right\|_{2} /\left\|\mathbf{B}_{C} \mathbf{s}_{C}\right\|_{2}, \mathbf{S}_{C}, \mathbf{s}_{C}$ and $\mathbf{Q}_{C}, \mathbf{q}_{C}$ are the noise-free and noise components of $\mathbf{X}_{C}=\left[\mathbf{X}_{r 1}^{T}, \cdots, \mathbf{X}_{r D}^{T}, \mathbf{X}_{i 1}^{T}, \cdots, \mathbf{X}_{i D}^{T}\right]^{T}, \mathbf{b}_{C}=\left[\mathbf{b}_{r 1}^{T}, \cdots, \mathbf{b}_{r D}^{T}, \mathbf{b}_{i 1}^{T}, \cdots, \mathbf{b}_{i D}^{T}\right]^{T}$, respectively, $\mathbf{B}_{C}^{T} \mathbf{B}_{C}=\mathbf{W}_{C}=\operatorname{blkdiag}\left(\mathbf{W}_{S}, \cdots, \mathbf{W}_{S}, \mathbf{W}_{S}, \cdots, \mathbf{W}_{S}\right)$. Then from (30), ignoring the difference in the conditioning of $\mathbf{B}_{C}$ and relative errors $\epsilon_{1}, \epsilon_{2}$ with different decimation factors, we can expect smaller relative error of LP coefficients occurs when the condition number of matrix $\mathbf{S}_{C}$ is lower.

It is difficult to estimate the condition number of $\mathbf{S}_{C}$ from the available but noisy data matrix $\mathbf{X}_{C}$, especially when $\mathbf{S}_{C}$ is ill-conditioned. Nevertheless, from [19], [20], we know that the ill-conditioning of $\mathbf{S}_{C}$ occurs from closely-spaced frequencies, and higher decimation factor, which separates adjacent angular frequencies more, tends to improve the conditioning of $\mathbf{S}_{C}$. Therefore, in practice, we select the maximum possible value, $M$, as the decimation factor to achieve frequency estimation. Although the optimum decimation factor, which minimizes the data matrix's condition number $k\left(\mathbf{S}_{C}\right)$ in the interval $[1, M]$, may have the advantage over the practical decimation factor in terms of threshold performance, they can both work well in moderate to high signal-to-noise ratio (SNR) conditions.

Now that the decimated sampling rate $f_{S}^{\prime}=f_{S} / D$ remains not lower than the base frequency $f_{B}$, it is straightforward that the angular frequency estimates are

$$
\hat{\omega}_{l}=\hat{\omega}_{D l} / D, \quad l=1,2, \cdots, L
$$

where $\hat{\omega}_{D l}$ are the phases of the $L$ roots of the following equation lying in the interval $(0, \pi)$ :

$$
\sum_{i=0}^{L-1} \hat{a}_{i}^{\prime}\left(z^{i}+z^{(2 L-i)}\right)+\hat{a}_{L}^{\prime} z^{L}=0 .
$$

\section{Performance Analysis}

\subsection{Frequency Resolution}

It is illustrated in the algorithm development that the sequence is decimated by a factor of $D=M$ prior to estimation, where $M$ is the ratio of the sampling rate to the base frequency. Therefore, a decimation factor of $M$ is selected so that the frequencies are separated by $M$ times. This means that in the OSWLS estimation, the oversampling will not lower down the frequency resolution capacity compared to the sampling at the base frequency.

\subsection{Computational Complexity}

In the OSWLS frequency estimation with decimator $D$, the main computational complexity (taking only the multiplications into account) in one iteration consists of four main parts from (29): (i) $D$ times of 
matrix multiplication of $\mathbf{X}_{r i}^{T} \mathbf{W}_{S} \mathbf{X}_{r i}$ and $\mathbf{X}_{i i}^{T} \mathbf{W}_{S} \mathbf{X}_{i i}$; (ii) $D$ times of matrix multiplication of $\mathbf{X}_{r i}^{T} \mathbf{W}_{S} \mathbf{b}_{r i}$ and $\mathbf{X}_{i i}^{T} \mathbf{W}_{S} \mathbf{b}_{i i}$; (iii) matrix inversion of $\left(\sum_{i=1}^{D} \mathbf{X}_{r i}^{T} \mathbf{W}_{S} \mathbf{X}_{r i}+\sum_{i=1}^{D} \mathbf{X}_{i i}^{T} \mathbf{W}_{S} \mathbf{X}_{i i}\right)^{-1}$; (iv) construction of the weighting matrix $\mathbf{W}_{S}$, which require FLOPs of about $\mathcal{O}\left(L \times N^{2} \times D^{-1}\right)$ totally. Therefore, the computational complexity is inversely proportional to the decimation factor $D$ approximately. However, when the sequence is decimated highly, a larger factor puts less effect on the computational saving in the primary part $\mathcal{O}(L \times$ $N^{2} \times D^{-1}$ ), while costs more in other minor parts. Thus, the computation is similar for large decimation factors.

\subsection{Estimation Accuracy}

In parameter estimation, minimum variance unbiased estimator is always desired, which means that the estimator is unbiased and its variance is minimum [23]. When the data length or SNR is sufficiently large, it is directly derived from [8] that the mean of the OSWLS LP coefficient estimate $\hat{\mathbf{a}}^{\prime}$ is unbiased, that is, $E\left\{\hat{\mathbf{a}}^{\prime}\right\} \approx \mathbf{a}^{\prime}$, and the covariance matrix $\operatorname{cov}\left(\hat{\mathbf{a}}^{\prime}\right)$ is approximated as

$$
\mathbf{C}_{\hat{\mathbf{a}}^{\prime}} \approx \frac{1}{2} \sigma_{q}^{2}\left(\mathbf{S}_{C}^{T} \mathbf{W}_{C} \mathbf{S}_{C}\right)^{-1}
$$

Accordingly, the estimation variance of the $l$-th decimated sinusoidal pole $z_{D l}=e^{j D \omega_{l}}$ is evaluated as (see Appendix)

$$
\operatorname{var}\left(\hat{z}_{D l}\right)=\frac{1}{|\beta|^{2}} \boldsymbol{\mu}^{T} \mathbf{C}_{\hat{\mathbf{a}}^{\prime}} \boldsymbol{\mu}
$$

where

$$
\begin{aligned}
& \boldsymbol{\mu}=\left[\begin{array}{llll}
2 \cos \left((L-1) D \omega_{l}\right) & \cdots & 2 \cos \left(D \omega_{l}\right) & 1
\end{array}\right]^{T}, \\
& \beta=2 L z_{D l}^{2 L-1}+\sum_{i=1}^{L-1}\left(i z_{D l}^{i-1}+(2 L-i) z_{D l}^{2 L-i-1}\right) a_{i}^{\prime}+L z_{D l}^{L-1} a_{L}^{\prime},
\end{aligned}
$$

and the variances of the angular frequency $\omega_{l}$ and signal frequency $f_{l}$ estimators are expressed as [24]

$$
\operatorname{var}\left(\hat{\omega}_{l}\right)=\frac{1}{D^{2}} \operatorname{var}\left(\hat{z}_{D l}\right)
$$

and

$$
\operatorname{var}\left(\hat{f}_{l}\right)=\frac{f_{S}^{2}}{4 \pi^{2} D^{2}} \operatorname{var}\left(\hat{z}_{D l}\right)
$$

Now that the OSWLS estimator is based on the iterative quadratic maximum likelihood (IQML) technique [25], it can provide optimum accuracy asymptotically, that is the theoretical variances of the frequency estimators (37), (38) are equal to CRLB when the data length $N$ is large enough. It is difficult to analyze the relationship between the decimation factor $D$ and the theoretical variances. However, from a heuristic viewpoint and (22) - (28), the estimation accuracy is relevant to the total number of the elements of the $D$ LP error vectors $\mathbf{e}_{i}, i=1, \cdots, D$, and more error elements participating in the frequency estimation provide better accuracy. For large data length $N$, this number keeps nearly constant for different decimation factors, 
and approximately equal to the data length $N$, which is the case in oversampling scenario. So we can regard the estimation accuracy irrelevant to the decimation factor $D$.

In the following, we explore the theoretical variance for the frequency estimation of a single sinusoid to validate the above analysis. Following [8], the variance for the LP coefficient $a_{1}^{\prime}=-2 \cos \left(D \omega_{1}\right)$, when $N \rightarrow \infty$, is derived as

$$
\begin{aligned}
\operatorname{var}\left(\hat{a}_{1}^{\prime}\right) & \approx \frac{1}{2} \sigma_{q}^{2}\left(\mathbf{S}_{C}^{T} \mathbf{W}_{C} \mathbf{S}_{C}\right)^{-1} \\
& \approx \frac{24 \sin ^{2}\left(\omega_{1}\right)}{R D\left(N^{\prime}-2\right)\left(\left(N^{\prime}-2\right)^{2}-1\right)} \\
& \approx \frac{24 \sin ^{2}\left(\omega_{1}\right)}{R D N^{\prime 3}} \\
& \approx \frac{24 D^{2} \sin ^{2}\left(\omega_{1}\right)}{R N^{3}}
\end{aligned}
$$

where $R=\alpha_{1}^{2} / \sigma_{q}^{2}$ is the SNR. Accordingly, the variances of $\hat{\omega}_{1}$ and $\hat{f}_{1}$ are [8]

$$
\operatorname{var}\left(\hat{\omega}_{1}\right)=\frac{6}{R N^{3}},
$$

and

$$
\operatorname{var}\left(\hat{f}_{1}\right)=\frac{3 f_{S}^{2}}{2 \pi^{2} R N^{3}},
$$

which are equal to the asymptotic CRLB for single-tone frequency estimation in white Gaussian noise [23]. From (40) - (41), it is also seen that for large $N$, the frequency variances are not dependent on the decimation factor $D$.

\section{Simulation Results}

Monte Carlo simulations were carried out to evaluate the oversampling frequency estimation performance of the proposed approach. The signal frequency estimation accuracy was evaluated using the mean squared error (MSE): $\operatorname{MSE}(x)=E\left\{(x-\hat{x})^{2}\right\}$. All the results provided were averages of 500 independent runs. We used the number of iterations as the stopping criterion in the OSWLS estimation, which was assigned as 15. And these simulations were conducted on a PC with an Intel(R) Core(TM) 2 Duo CPU T6400@ 2.00 GHz, with 2 GB of memory.

Before the performance evaluation, it is necessary to verify the estimation improvement with oversampling. This is observed by the CRLB [26] - [27] with respect to sampling rate for a fixed duration T. Fig. 1 shows the CRLB performance of two sinusoidal signals: single-tone (with $\alpha=1, f_{1}=1 \mathrm{~Hz}, \phi_{1}=1, f_{B}=$ $2.5 \mathrm{~Hz}$ ) and dual-tone ( $w i t h \alpha_{1}=1, f_{1}=0.9 \mathrm{~Hz}, \phi_{1}=1, \alpha_{2}=1, f_{2}=1 \mathrm{~Hz}, \phi_{2}=2, f_{B}=2.5 \mathrm{~Hz}$ ), respectively, when duration $T$ is $10 \mathrm{~s}$, and SNR is $20 \mathrm{~dB}$. Note that since the CRLB is proportional to the noise power $\sigma_{q}^{2}$ in the case of white Gaussian noise [18], the curves in Fig. 1 are similar for other SNR values.

It is shown in Fig. 1 that the CRLBs go down with the increase of sampling rate, but at a rate slower and slower. The CRLBs in both situations decrease by about $10 \mathrm{~dB}$ when the sampling rate is 10 times the 
base frequency $f_{B}$; while by additional $10 \mathrm{~dB}$ when the sampling rate is increased by further 10 times. This indicates that on one hand, it is reasonable to utilize oversampling to improve the estimation accuracy. On the other hand, its effect comes less when raising the sampling rate higher. Considering the computational complexity, it is suggested to oversample the signal at 10 times the base frequency.

Fig. 2 shows the MSE of the WLS with different sampling rates as well as their theoretical counterparts and CRLBs, for the above dual-tone signal. Now that the two tones are separated apart enough even in oversampling, and we only care for the asymptotic estimation accuracy here, we do not use decimation technique. From Fig. 2, it is seen that the WLS gives optimum estimation accuracy in multiple complex sinusoids, when SNR is high enough. In addition, it is also clear that the higher the sampling rate is, the more accurate the frequency estimation is, and the sampling rate 10 times the base frequency provides 10 dB accuracy improvement, as expected.

Now consider the single-tone sinusoidal signal: $s(n)=\alpha_{1} e^{j\left(2 \pi f_{1} n / f_{S}+\phi_{1}\right)}$, where $\alpha_{1}=1, f_{1}=1 \mathrm{~Hz}, \phi_{1}=$ $1, f_{B}=2.5 \mathrm{~Hz}, f_{S}=10 f_{B}=25 \mathrm{~Hz}, T=8 \mathrm{~s}$, and $N=T \cdot f_{S}=200$. Fig. 3 shows the MSE of the above signal with respect to $\mathrm{SNR}$, with $D=1,5,10$, as well as their theoretical counterpart and CRLBs. For comparison, the result of a decimative version of U-ESPRIT [28], [29] with $D=10$, is provided. It is seen that when SNR $\geq 2 \mathrm{~dB}$, the OSWLS estimates with different decimation factors agree well with their theoretical calculation of (41), which is equal to the optimum accuracy. Meanwhile, the estimation without decimation, that is when $D=1$, bears a poorer threshold SNR. By comparison, the U-ESPRIT method has a bit higher MSE than CRLB by about $1 \mathrm{~dB}$.

Next, consider the sinusoidal signal with two closely-spaced tones: $s(n)=\alpha_{1} e^{j\left(2 \pi f_{1} n / f_{S}+\phi_{1}\right)}+$ $\alpha_{2} e^{j\left(2 \pi f_{2} n / f_{S}+\phi_{2}\right)}$, where $\alpha_{1}=2, f_{1}=0.96 \mathrm{~Hz}, \phi_{1}=1, \alpha_{2}=1, f_{2}=1 \mathrm{~Hz}, \phi_{2}=2, f_{B}=2.5 \mathrm{~Hz}, f_{S}=$ $10 f_{B}=25 \mathrm{~Hz}, T=12 \mathrm{~s}$, and $N=T \cdot f_{S}=300$. Fig. 4 shows the condition number of the data matrix $\mathbf{S}_{C}$ with respect to the decimation factor $D$. It is observed that the conditioning of the data matrix forms an oblique $L$-shape curve, and has minimal value at the corner $D=7$. Compared with the data matrix's conditioning when $D=1$, the condition numbers for the practical factor $D=10$ and optimum one $D=7$ are comparable. Table 1 summarizes the estimation outcomes for different decimation factors when $\mathrm{SNR}=20 \mathrm{~dB}$. It is shown that the MSE values come close to the CRLB with $D=7$ and $D=10$, and the latter is more computationally attractive. From Table 1, we can also find that when the decimation factor is small, the frequency estimation fails, and there is no meaningful estimate.

Fig. 5 shows the MSE of the above signal with respect to $\mathrm{SNR}$, with $D=7,10$, as well as their theoretical counterparts and CRLBs. For comparison, the results of the WLS and U-ESPRIT estimators are also provided. It is observed that all estimators can resolve the two adjacent frequencies when the SNR is high enough, and their asymptotic MSE values are equal to the CRLB. Nevertheless, the OSWLS estimators bear the threshold SNR advantage over the WLS estimator by more than $10 \mathrm{~dB}$. Although the U-ESPRIT estimator also bears high frequency resolution, it is not an optimum estimator, and there is about $2 \mathrm{~dB}$ gap between its MSE and CRLB. Note that from (a), the OSWLS estimators with $D=7$ and 10 have similar threshold SNR. So it is feasible to select the maximum possible decimator as the practical one. In addition, 
the MSE is less than the CRLB for the WLS estimator in (a) and the OSWLS one with $D=10$ in (b) when SNR is less than $20 \mathrm{~dB}$ and $10 \mathrm{~dB}$, respectively. Thus, the proposed estimator will provide biased estimates when the SNR is small enough.

In Fig. 6, we still consider a dual-tone sinusoidal signal when SNR $=20 \mathrm{~dB}$, and show the MSE for different $f_{2}$ while $f_{1}=0.625 \mathrm{~Hz}$ is fixed. $\alpha_{1}, \alpha_{2}$ are both set as 1 , and the remaining signal parameters are the same as that in the last numerical example. It is seen that the OSWLS estimator with different decimation factors can all resolve the two frequencies $f_{1}$ and $f_{2}$ when they are well separated. When these two frequencies come close, a higher decimation factor will provide a higher frequency resolution. Here, the OSWLS estimators with $D=4$ and $D=7$ can work with minimum frequency separations of $\Delta f=0.042 \mathrm{~Hz}$ and $\Delta f=0.036 \mathrm{~Hz}$, respectively, while for $D=10$, the accuracy loss (that is the gap between MSE and CRLB) keeps within $6 \mathrm{~dB}$ until $\Delta f$ becomes as small as $0.032 \mathrm{~Hz}$. Note that the MSE for $D=10$ increases more slowly with the decrease of frequency spacing than that for $D=4$ and $D=7$.

Fig. 7 shows the comparison of the OSWLS estimator with the WLS and U-ESPRIT estimators. The sinusoidal signal consists of three tones: $s(n)=\alpha_{1} e^{j\left(2 \pi f_{1} n / f_{S}+\phi_{1}\right)}+\alpha_{2} e^{j\left(2 \pi f_{2} n / f_{S}+\phi_{2}\right)}+\alpha_{3} e^{j\left(2 \pi f_{3} n / f_{S}+\phi_{3}\right)}$, where $\alpha_{1}=1, f_{1}=0.90 \mathrm{~Hz}, \phi_{1}=1, \alpha_{2}=1, f_{2}=0.95 \mathrm{~Hz}, \phi_{2}=2, \alpha_{3}=1, f_{3}=1 \mathrm{~Hz}, \phi_{3}=3, f_{B}=$ $2.5 \mathrm{~Hz}, f_{S}=10 f_{B}=25 \mathrm{~Hz}, T=20 \mathrm{~s}$, and $N=T \cdot f_{S}=500$. For the sake of simplicity, here we use the average MSE of frequency estimates for the performance evaluation. The optimum decimation factor for the OSWLS estimator is $D=8$. From this figure, we can find that the OSWLS estimators can resolve the sinusoidal tones, and give optimum estimation when SNR $\geq 10 \mathrm{~dB}$. By comparison, the WLS estimator cannot resolve the closely-spaced sinusoidal tones in the considered SNR range. Moreover, the U-ESPRIT estimator is not optimum, and there is about $2.5 \mathrm{~dB}$ gap between its MSE and CRLB. Note that for the OSWLS estimator, the practical decimation factor $D=10$ performs similarly to the optimum decimation factor $D=8$, and these two factors have the same threshold SNR, that is $10 \mathrm{~dB}$.

\section{Summary}

The OSWLS approach is proposed for the oversampling parameter estimation of multiple complex sinusoids. First, the WLS approach in [8] is extended to the complex situation. To overcome the problems of frequency resolution and computational complexity in oversampling spectral estimation, decimation technique is utilized. Performance analysis of the OSWLS scheme is given, including the frequency resolution, computational complexity, and estimation variance. Simulation results show that oversampling technique gives better accuracy compared to the base-frequency sampling, and attains the optimum MSE performance when the SNR is sufficiently large.

The angular frequencies of sinusoidal signals are assumed to range in $(0, \pi)$. If the signal is band-limited instead, further decimation is allowed even if the frequency aliasing occurs. We can recover the original frequencies on the condition that the signal's center frequency is known approximately. 


\section{Appendix - Derivation of Estimation Variance of Sinusoidal Poles}

Since $\alpha\left(z_{D l}\right)=\sum_{i=0}^{L-1} a_{i}^{\prime}\left(z_{D l}^{i}+z_{D l}^{2 L-i}\right)+a_{L}^{\prime} z_{D l}^{L}=0$,

$$
\frac{\partial \alpha\left(z_{D l}\right)}{\partial z_{D l}} d z_{D l}+\frac{\partial \alpha\left(z_{D l}\right)}{\partial a_{1}^{\prime}} d a_{1}^{\prime}+\cdots+\frac{\partial \alpha\left(z_{D l}\right)}{\partial a_{L}^{\prime}} d a_{L}^{\prime}=0, \quad i=1,2, \cdots, L,
$$

or

$$
\begin{aligned}
& \left(2 L z_{D l}^{2 L-1}+\sum_{i=1}^{L-1}\left(i z_{D l}^{i-1}+(2 L-i) z_{D l}^{2 L-i-1}\right) a_{i}^{\prime}+L z_{D l}^{L-1} a_{L}^{\prime}\right) d z_{D l} \\
& +\left(z_{D l}^{1}+z_{D l}^{2 L-1}\right) d a_{1}^{\prime}+\cdots+\left(z_{D l}^{L-1}+z_{D l}^{L+1}\right) d a_{L-1}^{\prime}+z_{D l}^{L} d a_{L}^{\prime}=0 .
\end{aligned}
$$

Thus,

$$
\begin{aligned}
& \Delta z_{D l}=-\frac{\left(z_{D l}^{1}+z_{D l}^{2 L-1}\right) d a_{1}^{\prime}+\cdots+\left(z_{D l}^{L-1}+z_{D l}^{L+1}\right) d a_{L-1}^{\prime}+z_{D l}^{L} d a_{L}^{\prime}}{2 L z_{D l}^{2 L-1}+\sum_{i=1}^{L-1}\left(i z_{D l}^{i-1}+(2 L-i) z_{D l}^{2 L-i-1}\right) a_{i}^{\prime}+L z_{D l}^{L-1} a_{L}^{\prime}}+O \\
& \approx-\frac{\left[\begin{array}{cccc}
z_{D l}^{1}+z_{D l}^{2 L-1} & \cdots & z_{D l}^{L-1}+z_{D l}^{L+1} & z_{D l}^{L}
\end{array}\right]}{2 L z_{D l}^{2 L-1}+\sum_{i=1}^{L-1}\left(i z_{D l}^{i-1}+(2 L-i) z_{D l}^{2 L-i-1}\right) a_{i}^{\prime}+L z_{D l}^{L-1} a_{L}^{\prime}} \Delta \mathbf{a}^{\prime}
\end{aligned}
$$

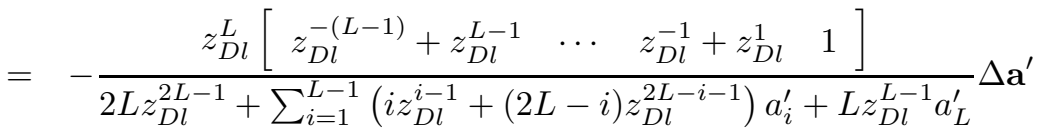

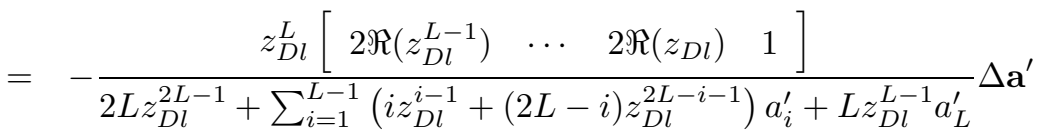

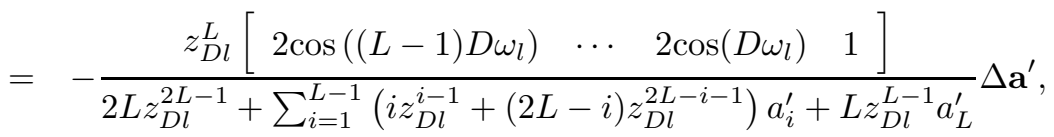

and

$$
\operatorname{var}\left(\hat{z}_{D l}\right)=E\left\{\left|\Delta z_{D l}\right|^{2}\right\}=\frac{1}{|\beta|^{2}} \boldsymbol{\mu}^{T} \mathbf{C}_{\hat{\mathbf{a}}^{\prime}} \boldsymbol{\mu}
$$

which completes the proof.

\section{References}

[1] P. Stoica and R. Moses, Spectral Analysis of Signals, Upper Saddle River, NJ: Pearson Prentice-Hall, 2005

[2] D. C. Rife and R. R. Boorstyn, "Multiple tone parameter estimation from discrete-time observations," Bell Syst. Tech. J., pp.1389-1410, Nov. 1976

[3] P. Stoica and A. Nehorai, "Statistical analysis of two non-linear least-squares estimators of sine waves parameters in the colored noise," in Proc. ICASSP, vol.4, pp.2408-2411, Apr. 11-14, 1988, New York, New York, USA

[4] Y. T. Chan and R. P. Langford, "Spectral estimation via the high-order Yule-Walker equations," IEEE Transactions on Acoustics, Speech and Signal Processing, vol.30, no.5, pp.689-698, Oct. 1982 
[5] M.D. Rahman and K.-B. Yu, "Total least squares approach for frequency estimation using linear prediction," IEEE Transactions on Acoustics, Speech and Signal Processing, vol.35, no.10, pp.1440-1454, Oct. 1987

[6] T.-H. Li and B. Kedem, "Iterative filtering for multiple frequency estimation," IEEE Transactions on Signal Processing, vol.42, no.5, pp.1120-1132, May 1994

[7] B. Porat, Digital Processing of Random Signals: Theory and Methods, Englewood Cliffs, NJ: Prentice-Hall, 1994

[8] H. C. So, K. W. Chan, Y. T. Chan and K. C. Ho, "Linear prediction approach for efficient frequency estimation of multiple real sinusoids: algorithms and analyses," IEEE Transactions on Signal Processing, vol.53, no.7, pp.2290-2305, July 2005

[9] A. Host-Madsen and P. Handel, "Effects of sampling and quantization on single-tone frequency estimation," IEEE Transactions on Signal Processing, vol.48, no.3, pp.650-662, Mar. 2000

[10] D. Li, X. Liu and K. Zhang, "Study on oversampling gain," in Proc. IEEE ICSP, pp.70-73, Oct. 24-28, 2010, Beijing, China

[11] H. Cramer, Mathematical Methods of Statistics, Princeton, N.J.: Princeton University Press, 1974

[12] P. Stoica, H. Li and J. Li, "Amplitude estimation of sinusoidal signals: survey, new results, and an application," IEEE Transactions on Signal Processing, vol.48, no.2, pp.338-352, Feb. 2000

[13] Y. Bresler and A. Macovski, "Exact maximum likelihood parameter estimation of superimposed exponential signals in noise," IEEE Transactions on Acoustics, Speech and Signal Processing, vol.34, no.5, pp.1081-1089, Oct. 1986

[14] Y. Hua, "The most efficient implementation of the IQML algorithm," IEEE Transactions on Signal Processing, vol.42, no.8, pp.2203-2204, Aug. 1994

[15] T. Soderstrom and P. Stoica, System Identification, Englewood Cliffs, N.J.: Prentice-Hall International, 1989

[16] C. Magi, T. Backstrom and P. Alku, "Simple proofs of root locations of two symmetric linear prediction models," Signal Processing, vol.88, no.7, pp.1894-1897, July 2008

[17] Y. T. Chan, J. M. M. Lavoie and J. B. Plant, "A parameter estimation approach to estimation of frequencies of sinusoids," IEEE Transactions on Acoustics, Speech and Signal Processing, vol.29, no.2, pp.214-219, Apr. 1981

[18] P. Stoica, A. Jakobsson and J. Li, "Cisoid parameter estimation in the colored noise case: asymptotic CramérRao bound, maximum likelihood, and nonlinear least-squares," IEEE Transactions on Signal Processing, vol.45, no.8, pp.2048-2059, Aug. 1997

[19] E. Fogel and M. Villalba, "On the sampling rate issue in spectral analysis," in Proc. IEEE ICASSP, vol.8, pp.1434-1437, Apr. 14-16, 1983, Boston, Massachusetts, USA

[20] P. Stoica and A. Nehorai, "MUSIC, maximum likelihood, and Cramér-Rao bound," IEEE Transactions on Acoustics, Speech and Signal Processing, vol.37, no.5, pp.720-741, May 1989

[21] D. W. Lewis, Matrix Theory, Singapore: World Scientific, 1991 
[22] J. K. Merikoski and R. Kumar, "Inequalities for spreads of matrix sums and products," Applied Mathematics E-Notes, vol.4, pp.150-159, 2004

[23] S. M. Kay, Fundamentals of Statistical Signal Processing: Estimation Theory, Englewood Cliffs, N.J.: PTR Prentice-Hall, 1993

[24] Y.-X. Yao and S. M. Pandit, "Variance of least squares estimators for a damped sinusoidal process," IEEE Transactions on Signal Processing, vol.42, no.11, pp.3016-3025, Nov. 1994

[25] Y. Hua, A. B. Gershman and Q. Cheng, High-Resolution and Robust Signal Processing, New York: Marcel Dekker, 2004

[26] G. Chardon and L. Daudet, "Optimal subsampling of multichannel damped sinusoids," in Proc. IEEE Sensor Array and Multichannel Signal Processing Workshop (SAM), pp.25-28, Oct. 4-7, 2010, Jerusalem, Israel

[27] S. Trittler and F. A. Hamprecht, "Near optimum sampling design and an efficient algorithm for single tone frequency estimation," Digital Signal Processing, vol.19, no.4, pp.628-639, July 2009

[28] M. Haardt and J. A. Nossek, "Unitary ESPRIT: how to obtain increased estimation accuracy with a reduced computational burden," IEEE Transactions on Signal Processing, vol.43, no.5, pp.1232-1242, May 1995

[29] G. Morren, P. Lemmerling and S. Van Huffel, "Decimative subspace-based parameter estimation techniques," Signal Processing, vol.83, no.5, pp.1025-1033, May 2003

\begin{tabular}{|c|c|c|c|c|c|}
\hline \multirow{3}{*}{$D$} & \multirow{2}{*}{\multicolumn{2}{|c|}{$\begin{array}{c}\text { Frequency } 1(0.96 \mathrm{~Hz}) \\
\text { CRLB: }-61.40 \mathrm{~dB}\end{array}$}} & \multirow{2}{*}{\multicolumn{2}{|c|}{$\begin{array}{l}\text { Frequency } 2(1 \mathrm{~Hz}) \\
\text { CRLB: }-55.38 \mathrm{~dB}\end{array}$}} & \multirow{3}{*}{ CPU Time (ms) } \\
\hline & & & & & \\
\hline & Mean $(\mathrm{Hz})$ & Variance $(\mathrm{dB})$ & Mean $(\mathrm{Hz})$ & Variance $(\mathrm{dB})$ & \\
\hline 1 & 0.9504 & -71.06 & 5.6248 & -2.33 & 6491 \\
\hline 2 & 0.9594 & -54.10 & 1.0095 & -26.45 & 875 \\
\hline 3 & 0.9600 & -61.67 & 1.0002 & -55.48 & 287 \\
\hline 4 & 0.9598 & -61.36 & 1.0006 & -55.16 & 134 \\
\hline 5 & 0.9598 & -61.64 & 1.0005 & -55.49 & 78 \\
\hline 6 & 0.9597 & -61.87 & 1.0006 & -55.28 & 53 \\
\hline 7 & 0.9596 & -61.97 & 1.0008 & -55.81 & 69 \\
\hline 8 & 0.9596 & -61.48 & 1.0008 & -55.14 & 57 \\
\hline 9 & 0.9596 & -61.63 & 1.0007 & -55.25 & 43 \\
\hline 10 & 0.9596 & -61.40 & 1.0009 & -55.40 & 23 \\
\hline
\end{tabular}

Table 1: Estimated values for dual-tone sinusoidal signal. 


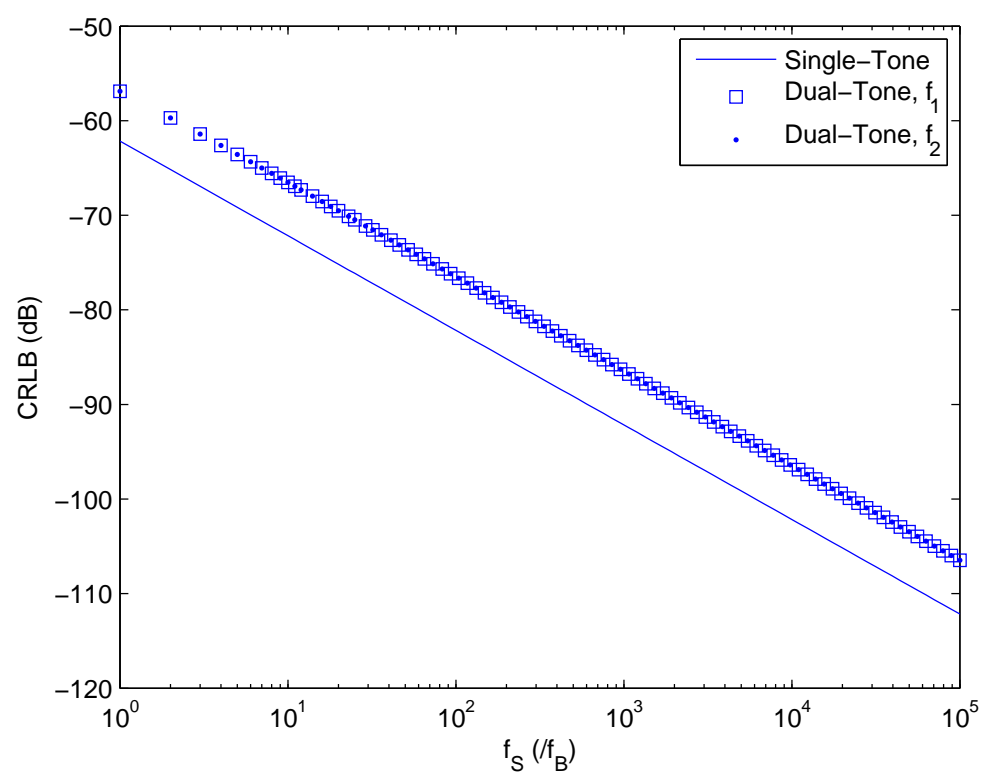

Figure 1: Relationship between CRLB and sampling rate. 


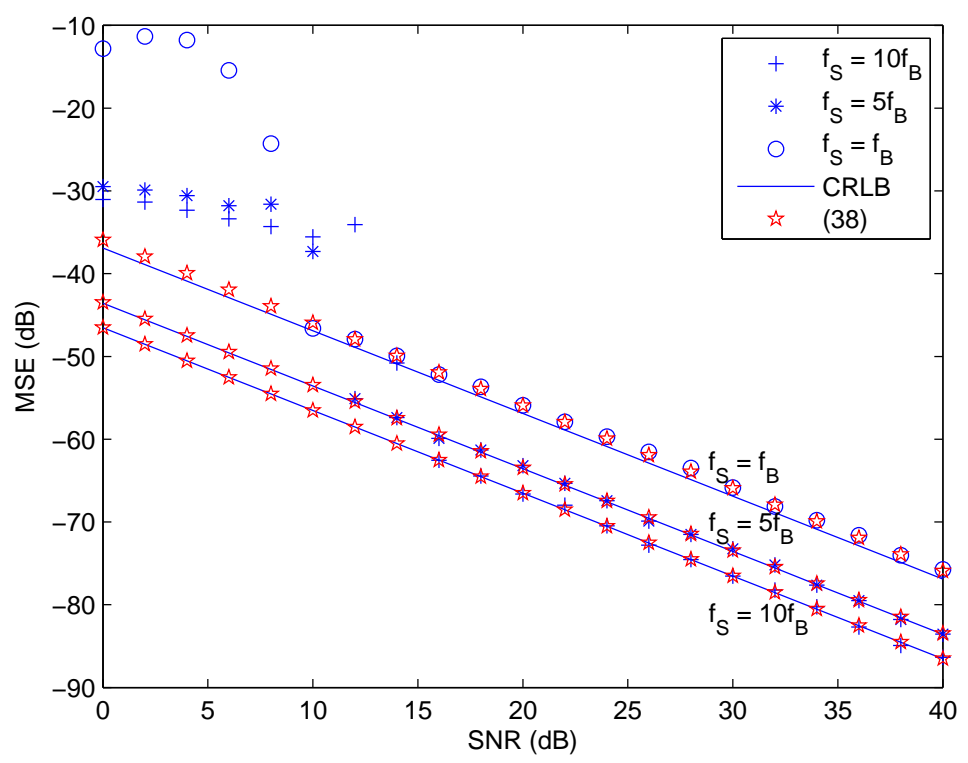

(a)

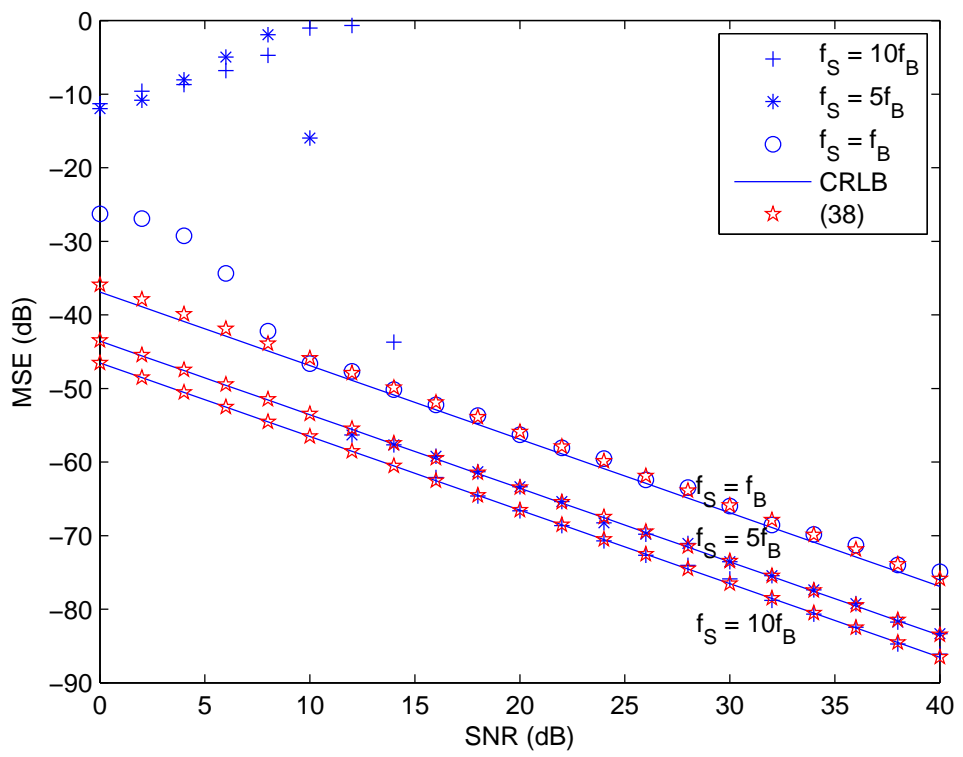

(b)

Figure 2: Dual-tone frequency estimation with sampling rates $f_{S}=f_{B}, 5 f_{B}, 10 f_{B}$. (a): $f_{1}$, (b): $f_{2}$. 


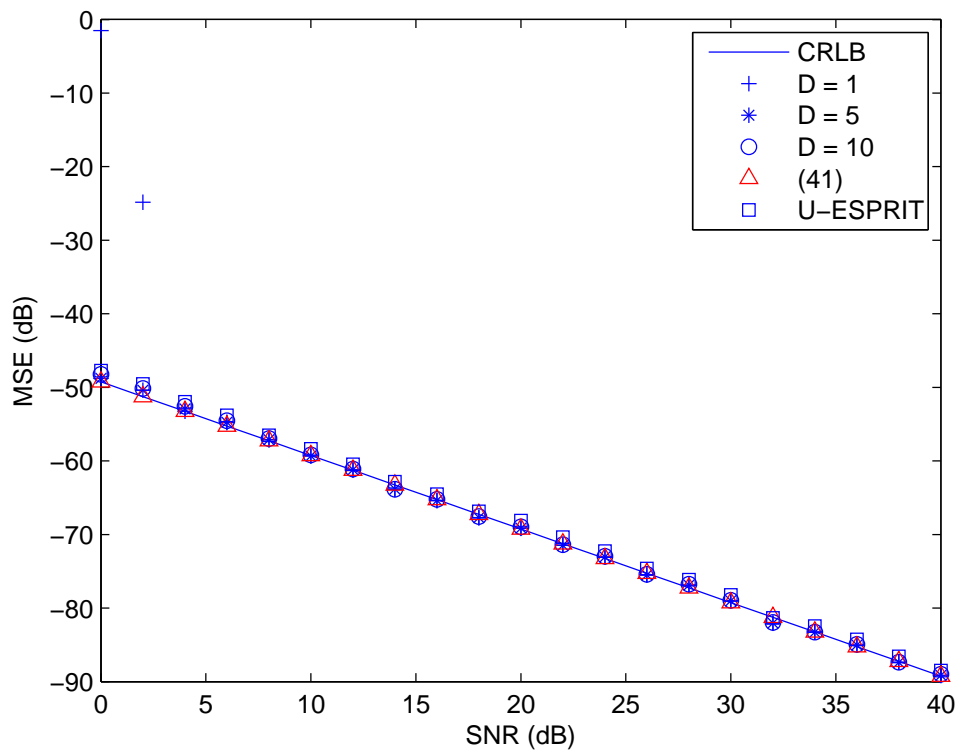

Figure 3: Single-tone frequency estimation of the OSWLS, WLS, U-ESPRIT estimators.

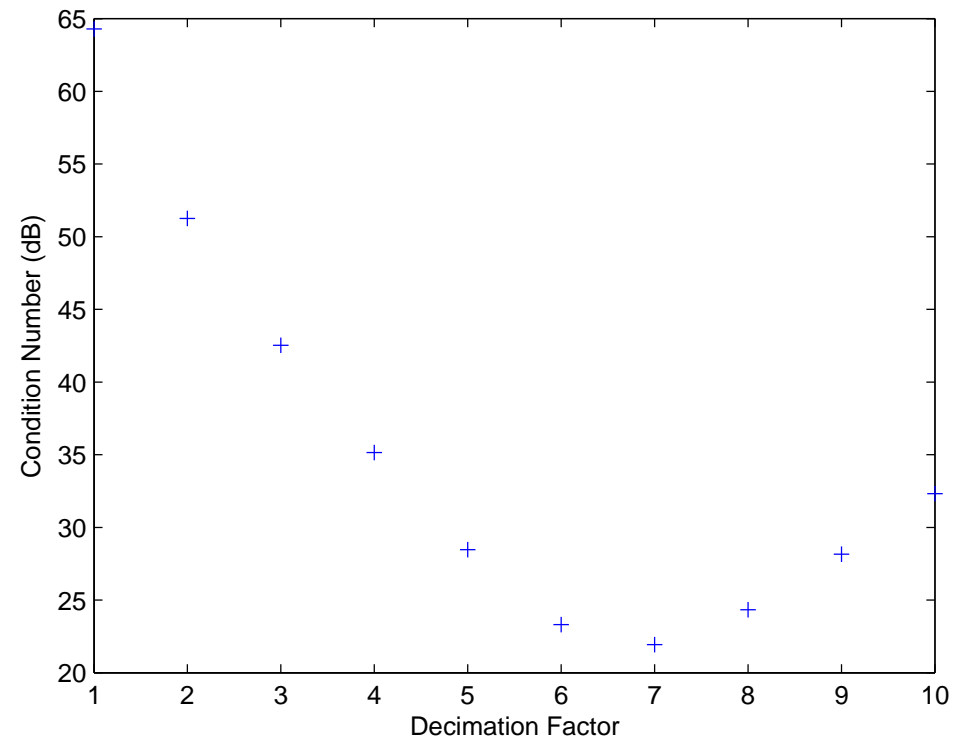

Figure 4: Condition number of data matrix versus decimation factor. 


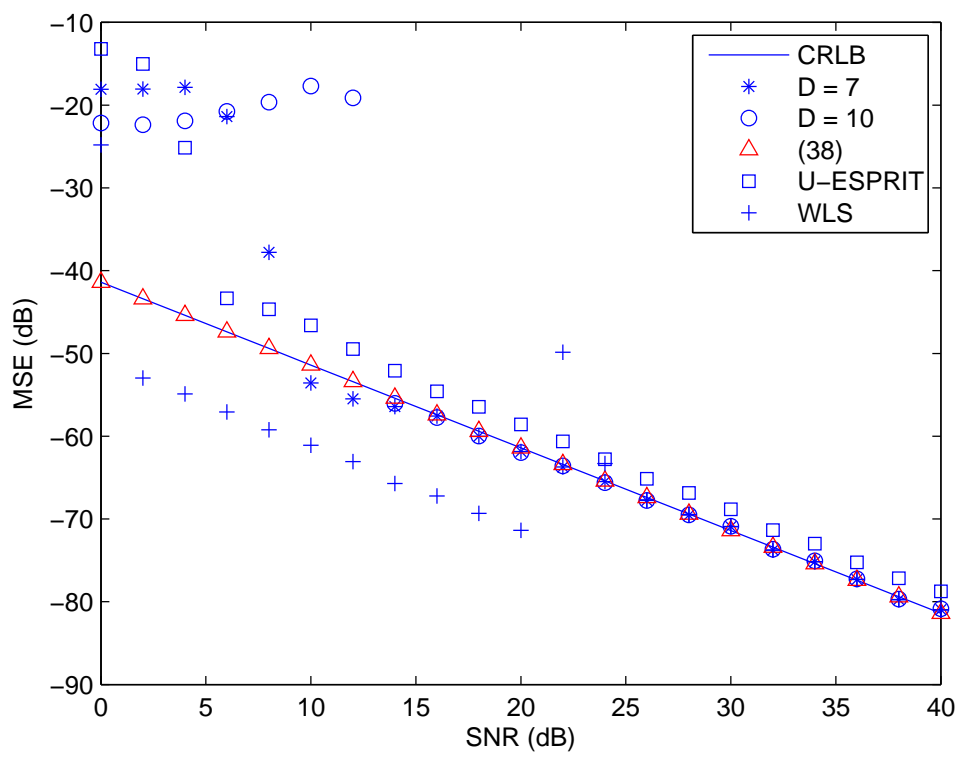

(a)

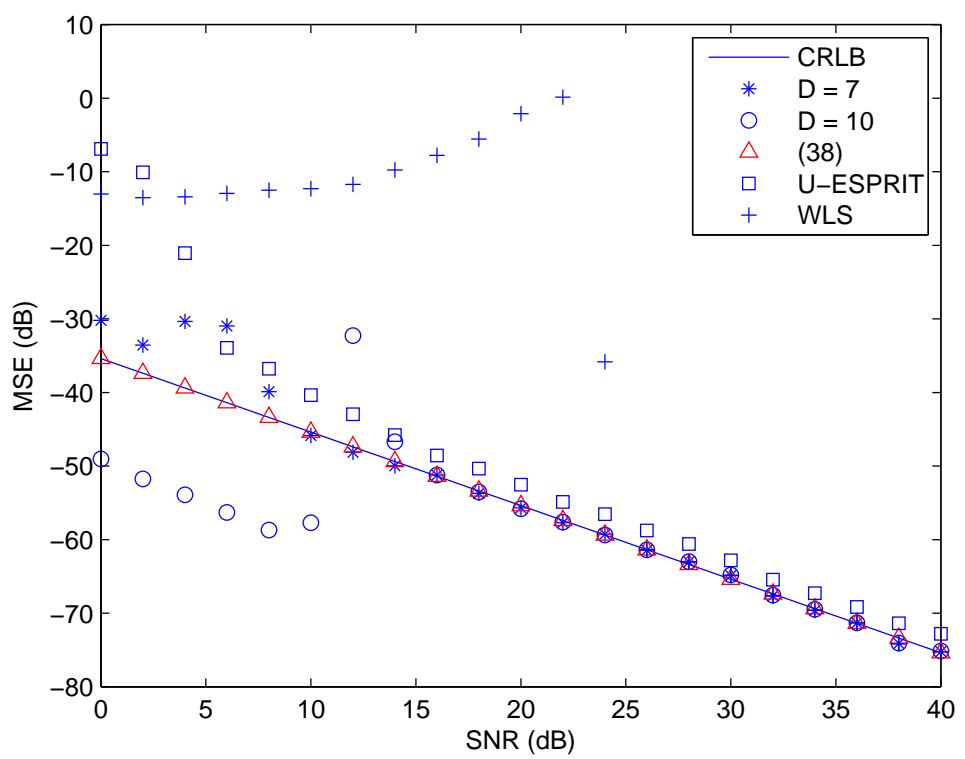

(b)

Figure 5: Dual-tone frequency estimation of the OSWLS, WLS, U-ESPRIT estimators. (a): $f_{1},(\mathrm{~b}): f_{2}$. 


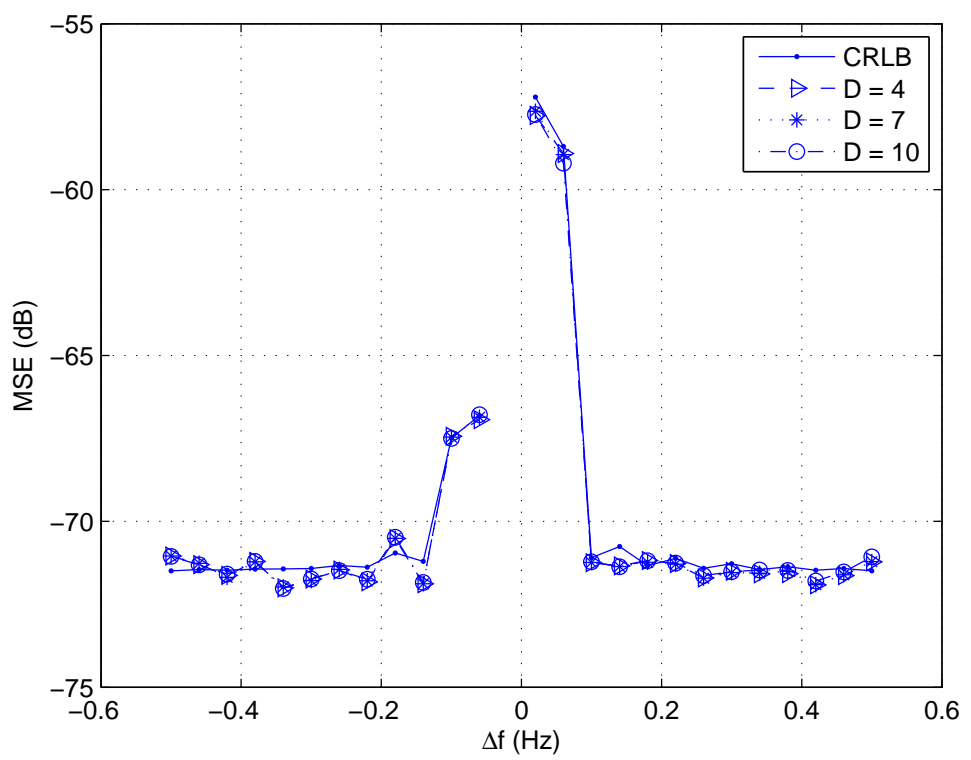

(a)

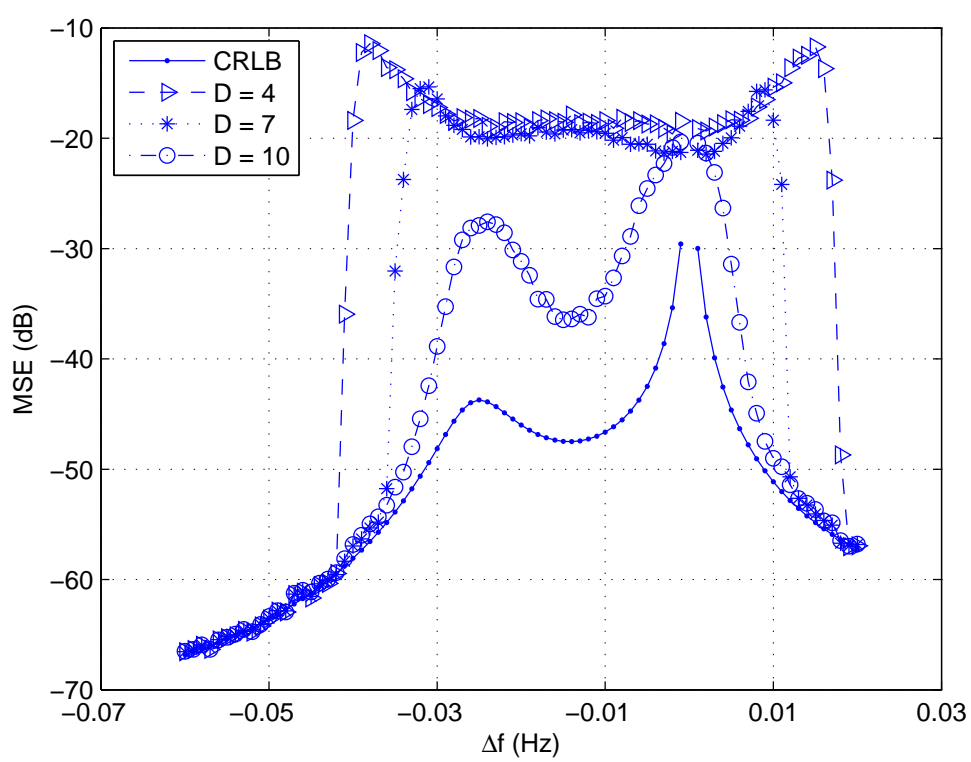

(b)

Figure 6: Dual-tone frequency estimation MSE for $f_{2}$ versus $\Delta f$. (a): Large frequency spacing, (b): Small frequency spacing. 


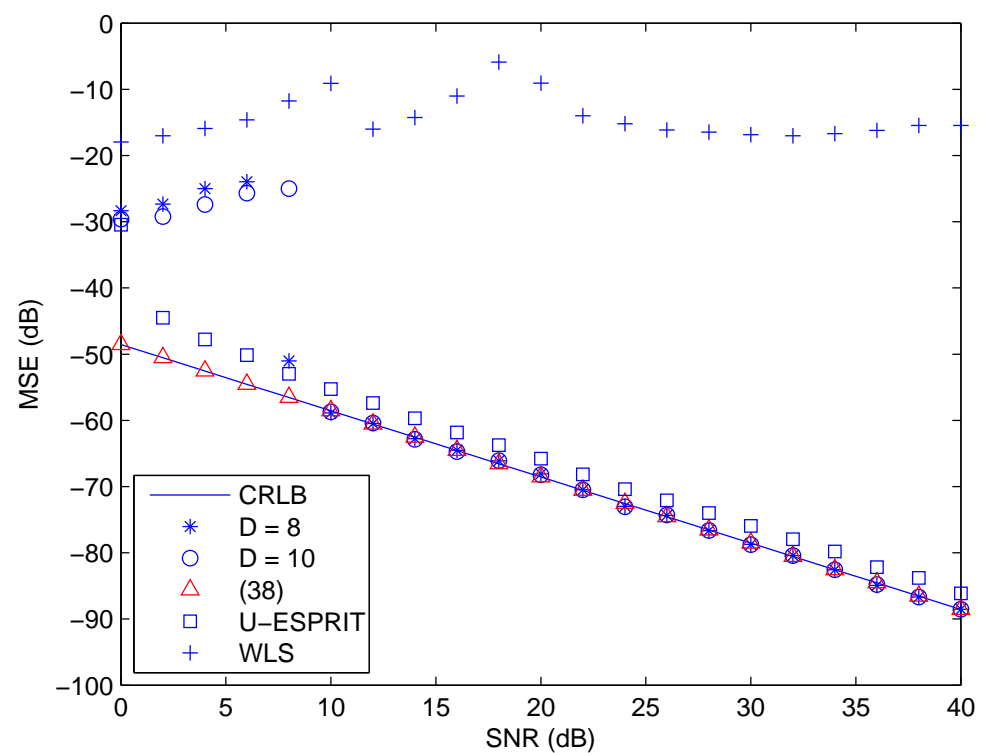

Figure 7: Triple-tone frequency estimation of the OSWLS, WLS, U-ESPRIT estimators. 